\title{
Employing Exfoliated Graphite as Novel Support Material for Heterogeneous Model Catalysts
}

Moritz Wolf, Nico Fischer, Michael Claeys

Submitted date: 03/05/2019 - Posted date: 06/05/2019

Licence: CC BY-NC-ND 4.0

Citation information: Wolf, Moritz; Fischer, Nico; Claeys, Michael (2019): Employing Exfoliated Graphite as Novel Support Material for Heterogeneous Model Catalysts. ChemRxiv. Preprint.

The inert nature of graphitic samples allows for characterisation of rather isolated supported nanoparticles in model catalysts, as long as sufficiently large inter-particle distances are obtained. However, the low surface area of graphite and the little interaction with nanoparticles result in a challenging application of conventional preparation routes in practice. In the present study, a set of graphitic carbon materials was characterised in order to identify potential support materials for the preparation of model catalyst systems. Various sizes of well-defined $\mathrm{Co}_{3} \mathrm{O}_{4}$ nanoparticles were synthesised separately and supported onto exfoliated graphite powder, that is graphite after solvent-assisted exfoliation via ultrasonication resulting in thinner flakes with increased specific surface area. The developed model catalysts are ideally suited for sintering studies of isolated nano-sized cobaltous particles as the graphitic support material does not provide distinct metal-support interaction. Furthermore, the differently sized cobaltous particles in the various model systems render possible studies on structural dependencies of activity, selectivity, and deactivation in cobalt oxide or cobalt catalysed reactions.

File list (2)

Wolf et al. Preprint.pdf (1.45 MiB)

view on ChemRxiv - download file

Wolf et al. Supporting material - Preprint.pdf (785.37 KiB)

view on ChemRxiv - download file 


\title{
Employing exfoliated graphite as novel support material for heterogeneous model catalysts
}

\author{
Moritz Wolf, ${ }^{\dagger, 1}$ Nico Fischer, ${ }^{\dagger}$ and Michael Claeys ${ }^{*}, \dagger$ \\ ${ }^{\dagger}$ Catalysis Institute and DST-NRF Centre of Excellence in Catalysis $c^{*}$ change, Department \\ of Chemical Engineering, University of Cape Town, Rondebosch 7701, South Africa. \\ *Corresponding author: Email michael.claeys@uct.ac.za
}

\begin{abstract}
The inert nature of graphitic samples allows for characterisation of rather isolated supported nanoparticles in model catalysts, as long as sufficiently large inter-particle distances are obtained. However, the low surface area of graphite and the little interaction with nanoparticles result in a challenging application of conventional preparation routes in practice. In the present study, a set of graphitic carbon materials was characterised in order to identify potential support materials for the preparation of model catalyst systems. Various sizes of well-defined $\mathrm{Co}_{3} \mathrm{O}_{4}$ nanoparticles were synthesised separately and supported onto exfoliated graphite powder, that is graphite after solvent-assisted exfoliation via ultrasonication resulting in thinner flakes with increased specific surface area. The developed model catalysts are ideally suited for sintering studies of isolated nano-sized cobaltous particles as the graphitic support material does not provide distinct metal-support interaction. Furthermore, the differently sized cobaltous particles in the various model systems render possible studies on structural dependencies of activity, selectivity, and deactivation in cobalt oxide or cobalt catalysed reactions.
\end{abstract}

\section{Introduction}

Traditional support materials have mostly been regarded as inert, but more recently even common metal oxide carriers have been shown to exhibit a strong effect on the catalytic behaviour ${ }^{1-4}$ and on the stability of the active phase specifically in the case of nano-sized material. ${ }^{5-9}$ These supports typically anchor the nanoparticles via metalsupport interactions sometimes even forming mixed phases. ${ }^{10-14}$ On the one hand, such an interaction is desired as it increases the stability of the nanoparticles under reaction

\footnotetext{
${ }^{1}$ Present address: Institute of Chemical Reaction Engineering, University of Erlangen-Nuremberg, 91058 Erlangen, Germany
} 
conditions, e.g. against sintering, on the other hand it is typically associated with a loss in active phase. Pristine carbon does not exhibit such stabilising properties, but may be functionalised with oxygen or nitrogen containing groups. ${ }^{15}$-17 However, even functionalised carbon shows a relative high chemical inertness except for the exposure to $\mathrm{O}_{2}$ at increased temperatures which results in combustion. ${ }^{17}$ Carbon supports are seldom applied in industry ${ }^{17,18}$ due to their lower mechanical strength compared to $\mathrm{Al}_{2} \mathrm{O}_{3}$ or $\mathrm{SiO}_{2}$ supports. ${ }^{18}$ However, their inert character makes carbon supports the carrier of choice for fundamental studies on the behaviour and stability of relatively isolated nanoparticles. ${ }^{19-22}$

Graphitic carbon materials comprise multiple layers of $\mathrm{sp}^{2}$-hybridised carbon atoms in two-dimensional planes. The atoms are arranged in the classic honeycomb like structure of graphene and the single layers are typically separated by 3.41 A and stacked in an $\mathrm{ABAB}$ order. ${ }^{23}$ The amount of stacked layers can be decreased via exfoliation techniques allowing the preparation of few-layer graphite or even graphene, a single two-dimensional plane of carbon atoms. ${ }^{24}$ The exfoliation of layered structures requires the overcoming of the weak van der Waals attractions between adjacent layers, ${ }^{23}$ which may be achieved for graphitic materials via immersion in solvents featuring a surface tension of approximately $40 \mathrm{~mJ} \mathrm{~m} \mathrm{~m}^{-2}$. Dispersion of graphitic material in such solvents results in a reduced interaction of adjacent layers. ${ }^{23,25}$ Exfoliation of immersed graphite may then be achieved by applying shearing forces via ball-milling ${ }^{26}$ or ultrasonication. ${ }^{27-}$ 29 The latter technique may yield up to $4 \mathrm{wt} . \%$ of graphene. ${ }^{28}$ However, pure few-layer graphite with up to $28 \%$ single-layer graphene may be achieved when applying a sizeselective centrifugation. ${ }^{27} \mathrm{~A}$ second, chemical approach decreases the van der Waals forces by expanding the distance between the layers allowing for simplified exfoliation. The chemical conversion of graphite to graphene via the preparation of intercalated graphite oxide according to a modification of Hummers' method ${ }^{30}$ with subsequent exfoliation into graphene oxide ${ }^{31}$ and reduction to graphene (commonly referred to as reduced graphene oxide $\mathrm{rGO})^{32}$ is one of the most widely applied methods due to its low cost in combination with considerable yields of the desired product. ${ }^{24,33,34}$ However, rGO differs strongly from pristine graphene obtained from aforementioned syntheses. The harsh oxidising and reducing conditions during the synthesis of rGO introduce considerable amounts of defects such as hydroxyl and epoxy groups on $\mathrm{sp}^{3}$-hybridised carbon on the basal plane disrupting its electronic properties. ${ }^{24,33}$ Defective sites originate from the incomplete reduction of oxidised tetrahedral $\mathrm{sp}^{3}$ to planar $\mathrm{sp}^{2}$ carbon atoms. ${ }^{33}$ However, modification of the synthesis may allow for the preparation of rGO with decreased concentrations of residual functional groups. ${ }^{35}$ Aside from mechanical exfoliation of graphitic precursors with the aid of suitable solvents or via intercalation of small molecules, a variety of chemical vapour deposition (CVD) techniques such as thermal CVD, plasma enhanced CVD, or a thermal decomposition on substrates are applied for the synthesis of graphene. ${ }^{17,24}$ 
The performance of carbon as catalyst or support material is determined by the surface chemistry of the carbon material. ${ }^{15}$ Functionalisation of carbon describes the introduction of functional groups such as carboxylic acids or carbonates on the carbon surface, ${ }^{15}$ which allow for an electronic interaction with nanoparticles, e.g. in heterogeneous catalysts. The interaction is more pronounced than for the pristine, clean surface and hence a higher stability against sintering, but also a lower reducibility, can be expected. Functionalisation techniques targeting the introduction of oxygen containing groups include acidic treatments in nitric, sulfuric, and/or phosphoric acid, as well as oxidative treatments with air, oxygen, nitrous oxide, potassium permanganate, or hydrogen peroxide. ${ }^{15,36-39}$ Functionalisation can also represent an artefact of the preparation of the carbon material, for example, aforementioned rGO contains various functional groups on the edges and the planar surfaces due to the harsh conditions applied during oxidation to graphitic oxide and reduction after exfoliation. ${ }^{24,33}$ In the present study pristine graphite is exfoliated and applied as novel support material in order to prepare rather isolated $\mathrm{Co}_{3} \mathrm{O}_{4}$ crystallites. The developed model system may allow for the characterisation of the physicochemical properties of isolated crystallites. For example, the stability of the crystallites may be analysed under conditions applied in catalytic processes with a minimised effect of the support material.

\section{Experimental}

\subsection{Chemicals}

Acetone, aqueous ammonia solution (25 wt.\%), and ethanol were purchased from Kimix (South Africa). Benzyl alcohol, cobalt(II) acetate tetrahydrate, synthetic graphite powder, and 1-methyl-2-pyrrolidone were obtained from Sigma-Aldrich. Kaylaw graphite powder is a chemically pure carbon material and was supplied by Electrochem (South Africa).

\subsection{Exfoliation of graphite}

Kaylaw graphite powder was exfoliated to increase its surface area. ${ }^{27}$ 1-Metyl-2pyrrolidinone (NMP) facilitates the exfoliation process as its surface energy is very close to the one of graphite. ${ }^{28}$ An amount of $3300 \mathrm{mg}$ graphite powder was sonicated in $1 \mathrm{~L}$ NMP for 12 h. ${ }^{28}$ The obtained suspension was centrifuged at $500 \mathrm{rpm}$ for $45 \mathrm{~min}$ to separate non-exfoliated and larger flakes of graphite. ${ }^{27}$ The exfoliated graphite (EG) in the remaining supernatant was collected via centrifugation at $7000 \mathrm{rpm}$ for $2 \mathrm{~h}$, subsequently washed several times with acetone, and dried at $80^{\circ} \mathrm{C}$ in an oven. 


\subsection{Synthesis of $\mathrm{CO}_{3} \mathrm{O}_{4}$ nanoparticles}

$\mathrm{Co}_{3} \mathrm{O}_{4}$ nanoparticles were synthesised via a surfactant-free, non-aqueous heat treatment of dissolved cobalt acetate in benzyl alcohol in the presence of ammonium hydroxide. ${ }^{9,14,22,40,41}$ Cobalt(II) acetate tetrahydrate was dissolved in benzyl alcohol under magnetic stirring at $500 \mathrm{rpm}$ in a round bottom flask (Table 1). After $2 \mathrm{~h}$ of stirring, a $25 \mathrm{wt} . \%$ aqueous ammonium hydroxide solution was added dropwise to the pink to purple solution forming a brown emulsion. Once the addition of ammonium hydroxide was completed, the flask including the stirrer bar was immediately transferred to a preheated oil bath of a rotary evaporation set-up and heated for a total of $3 \mathrm{~h}$ at $165{ }^{\circ} \mathrm{C}$. Air was bubbled through the reaction emulsion making use of the reduced pressure in the rotary flask (900 mbar) in order to ensure adequate mixing of the reaction emulsion. ${ }^{41}$

Table 1 Applied parameters in sol-gel syntheses of cobalt(II,III) oxide nanoparticles via the benzyl alcohol route.

\begin{tabular}{llll}
\hline Sample & Benzyl alcohol $/ \mathrm{mL}$ & $\mathrm{Co}\left(\mathrm{C}_{2} \mathrm{H}_{3} \mathrm{O}_{2}\right)_{2} \cdot 4 \mathrm{H}_{2} \mathrm{O} / \mathrm{mg}$ & $\mathrm{NH}_{4} \mathrm{OH}_{\mathrm{aq}}(25 \mathrm{wt} . \%) / \mathrm{mL}$ \\
\hline $\mathrm{Co} 2.8$ & 140 & 800 & 35 \\
$\mathrm{Co} 3.5$ & 140 & 400 & 35 \\
$\mathrm{Co}$ _4.9 & 70 & 1600 & 70 \\
$\mathrm{Co}$ _.6 & 140 & 3200 & 140 \\
\hline
\end{tabular}

After cooling-down to room temperature, the volume was tripled with diethyl ether and the mixture was centrifuged for 1 hour at $7000 \mathrm{rpm}$. The centrifugate containing the nanoparticles was re-dispersed in ethanol and washed at least three times with acetone until a clear supernatant was obtained..$^{40,41}$

\subsection{Supporting of nanoparticles}

$\mathrm{Co}_{3} \mathrm{O}_{4}$ crystallites were dispersed in ethanol in an ultrasonic bath until all nanoparticles were in dispersion $(>4 \mathrm{~h}$ ). Exfoliated graphite was sonicated for $4 \mathrm{~h}$ in ethanol after which the dispersion of $\mathrm{Co}_{3} \mathrm{O}_{4}$ nanoparticles in ethanol was added dropwise targeting the desired loading of metallic Co. After sonicating for another $4 \mathrm{~h}$, the dispersion was transferred to a rotary evaporator and further mixed for $1 \mathrm{~h}$ at 240 $\mathrm{rpm}$ and $80^{\circ} \mathrm{C}$. Subsequently, ethanol was evaporated from the parent sample at 462 mbar and the sample was dried at $80{ }^{\circ} \mathrm{C} .{ }^{9,14,22}$

\subsection{Characterisation}

Samples were analysed via transmission electron microscopy (TEM) in a Tecnai F20 microscope (Philips) equipped with a field emission gun and operated at $200 \mathrm{kV}$ 
(Gatan). Images were collected with a US4000 4kX4k CCD camera (Gatan). Nanoparticles were dispersed in ethanol via ultrasonication for $30 \mathrm{~min}$ prior to deposition onto carbon-coated copper grids. In contrast, supported nanoparticles were mixed with acetone and dispersed in the ultrasonication bath for $1 \mathrm{~min}$ to prevent separation of the nanoparticles from the support. Number-based size distributions were determined by measuring the size of over 500 nanoparticles using the open-source ImageJ 1.5la software package. ${ }^{42}$ The distribution of the nanoparticles was converted to a volume-based size distribution to allow for comparison with volume-based techniques such as X-ray diffraction (XRD). The number mean sizes $\left(d_{n, T E M}\right)$, the volume-mean sizes $\left(d_{V, T E M}\right)$, and the relative volume-based standard deviations $\left(\sigma_{V, T E M}\right)$ were calculated based on the measured sizes $d_{i}$ (Equations 1-3).

$$
\begin{aligned}
& d_{n, T E M}=\frac{\sum_{i=1}^{N} d_{i}}{N} \\
& d_{V, T E M}=\frac{\sum_{i=1}^{N} n_{i} \cdot d_{i}^{4}}{\sum_{i=1}^{N} n_{i} \cdot d_{i}^{3}} \\
& \sigma_{V, T E M}=\sqrt{\frac{\sum_{i=1}^{N}\left(n_{i} \cdot d_{i}^{3}\left(d_{i}-d_{V, T E M}\right)^{2}\right)}{\frac{N-1}{N} \sum_{i=1}^{N} n_{i} \cdot d_{i}^{3}}} / d_{V, T E M} \cdot 100 \%
\end{aligned}
$$

Where $N$ is the number of measured particles, and $n_{i}$ are the particular fractions.

Analysis of samples via Raman spectroscopy was conducted in an inVia Raman microscope (Renishaw) with a wavelength of the laser of $532 \mathrm{~nm}$. The Raman laser was fitted with a compact fibre optics probe (Renishaw). Five repetitions were taken at 40 $\mathrm{mW}$ laser power and an exposure time of $30 \mathrm{~s}$. The intensity ratio of the D and G band of graphitic samples allows for an estimation of the average in-plane crystallite size of the graphene layers (Equation 4$){ }^{43}$

$$
L_{a}=2.4 \cdot 10^{-10} \cdot \lambda^{4} \cdot\left(\frac{I_{D}}{I_{G}}\right)^{-1}
$$

Where $\lambda$ is the wavelength of the Raman laser and $I_{D}$ and $I_{G}$ are the intensities of the D band and $G$ band, respectively.

The surface areas of graphitic samples were analysed via physisorption according to the Brunauer-Emmett-Teller (BET) method. Physisorption was conducted using a TriStar II 3020 (Micromeritics) with $\mathrm{N}_{2}$ as analysis adsorptive and a degassing temperature of $200^{\circ} \mathrm{C}$.

Thermogravimetric analysis (TGA) of graphitic support material was conducted in a SDT 650 DSC/TGA simultaneous thermal analyser (TA Instruments). An amount of 8 to $12 \mathrm{mg}$ of material was loaded into a $90 \mu \mathrm{L}$ alumina crucible and heated from 50 to $900{ }^{\circ} \mathrm{C}$ at $5^{\circ} \mathrm{C} \mathrm{min}^{-1}$ in $\mathrm{N}_{2}$ to decompose functional groups associated with the surface of 
carbon. The measured weight loss then corresponds to such functional groups. ${ }^{15,44}$ In another analysis to determine the Co loading, the temperature was ramped from 120 to $900{ }^{\circ} \mathrm{C}$ at $10^{\circ} \mathrm{C} \mathrm{min}{ }^{-1}$ with a final holding time at $900{ }^{\circ} \mathrm{C}$ of $60 \mathrm{~min}$ in air. The residual mass corresponds to $\mathrm{CoO}$ being the stable cobalt oxide at $900{ }^{\circ} \mathrm{C},{ }^{45}$ which allows for the calculation of the Co loading.

XRD was conducted at $35 \mathrm{kV}$ and $40 \mathrm{~mA}$ in a D8 Advance X-ray diffractometer (Bruker AXS), equipped with a cobalt source $\left(\lambda_{K \alpha l}=0.178897 \mathrm{~nm}\right.$; slit width $\left.=1.0 \mathrm{~mm}\right)$ and a LYNXEYE XE position sensitive detector (Bruker AXS) from $20-120^{\circ}$ at a step size of $0.025^{\circ}$ with an exposure time of $1 \mathrm{~s}$ per step. Obtained XRD patterns were compared to reference patterns of the Powder Diffraction File of the International Centre for Diffraction Data (ICDD; PDF-2 Release 2008; ${ }^{46} \mathrm{Co}_{3} \mathrm{O}_{4}$ : 00-043-1003, cubic CoO: 01071-1178, fcc-Co: 00-015-0806, hcp-Co: 01-071-4239, graphite: 03-065-6212). Volumemean crystallite sizes and weight fractions of the particular phases were determined by Rietveld refinement of the XRD patterns (TOPAS 5, Bruker AXS) ${ }^{47}$ The instrumental line broadening was modelled via fitting the pattern of corundum. Diffraction line broadening analysis via the Scherrer equation ${ }^{48}\left(\mathrm{C}_{3} \mathrm{O}_{4}\right.$ : 311 diffraction) with correction for the instrumental line broadening and a shape factor of 0.9 was applied as a secondary technique to estimate the crystallite size of the as prepared nanoparticles via XRD. ${ }^{49}$

\section{Results and discussion}

\subsection{Characterisation of exfoliated graphite}

X-ray diffraction (XRD) patterns of graphite samples are dominated by the diffraction of the (002) plane at $30.9^{\circ}\left(\lambda_{C_{0}, K \alpha l}=1.78897 \AA\right.$; Figure la). This becomes even more pronounced for thick samples with an extended length perpendicular to the basal plane,$^{50}$ i.e. with high stacking numbers such as natural graphite flakes. The shape of the (002) reflection depends on the thickness of the graphite sample. Thinner graphite samples result in a broader peak with a maximum at lower diffraction angles due to the increased contribution of intercalated planes on top/bottom of graphitic flakes. ${ }^{50,51}$ XRD analysis of natural graphite flakes exhibits this dependency as the (002) reflection is bimodal indicating a wide range of stacking numbers (Figure $1 b$ ). Application of the Scherrer equation ${ }^{48}$ on the (002) diffraction line allows for an estimation of the average number of layers. ${ }^{33,51}$ In the present study, this method was extended to Rietveld refinement of the patterns from $28-33^{\circ}$ applying two separate graphite structures in order to obtain a range of the number of layers based on the calculated crystallite sizes (Table 2). As expected, size analysis of synthetic graphite powder (Sigma-Aldrich) and natural graphite flakes results in the former having less layers. Kaylaw graphite powder, a chemically pure carbon additive for lubricants, shows the broadest (002) reflection. 
Another indicator for the thickness of a graphite sample is the ratio of the reflection intensity of the interplanar distances (002) or (004) and the intraplanar (100) plane, which increases with the stacking number. This can be demonstrated by a comparison of the ratios for natural graphite flakes and commercially synthesised graphite powder (Table 2; Figure lc). The trend in the ratios is inversely proportional to the BET surface area (Figure S.1), which can be expected to increase with lower stacking numbers.

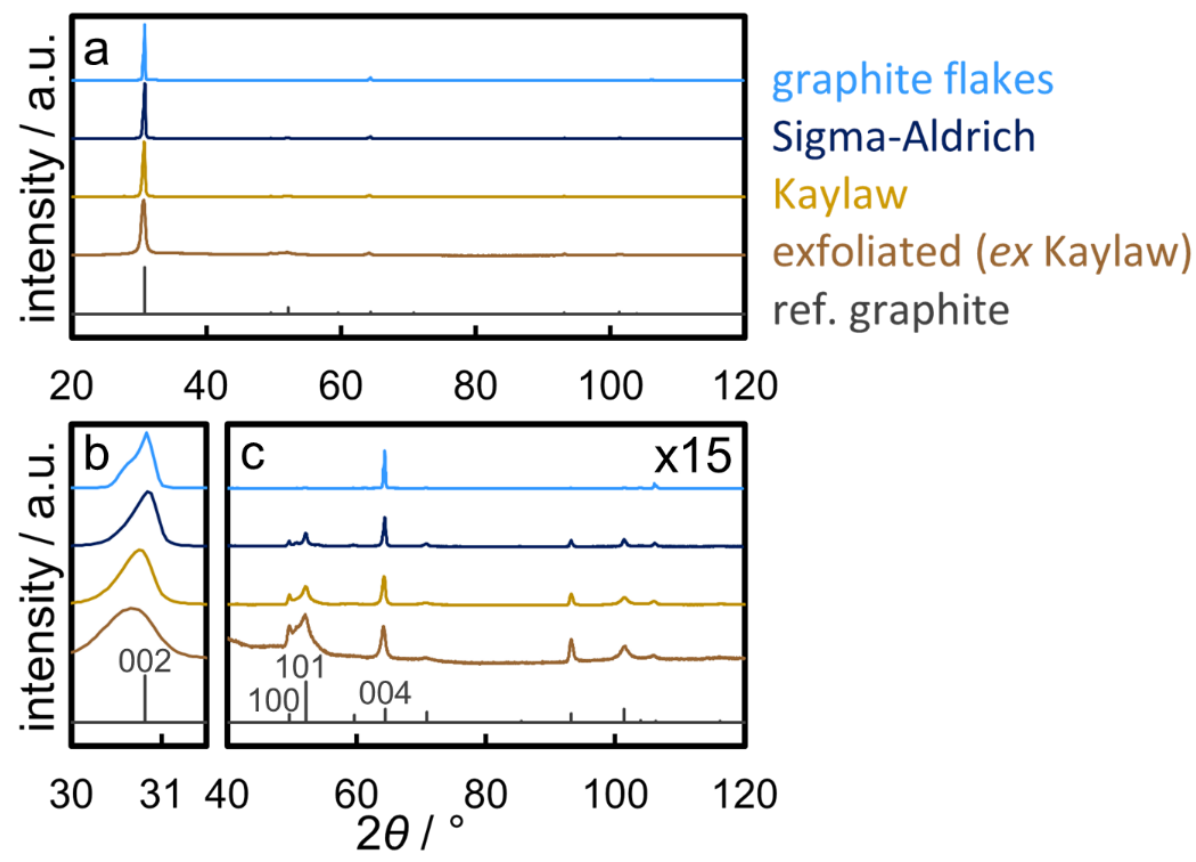

Figure 1 (a) Normalised X-ray diffraction patterns, (b) enlarged (002) reflections, and (c) the patterns above $40^{\circ}$ with 15 times increased intensities of graphitic materials with a reference pattern for graphite.

Table 2 Characteristics of reflections in X-ray diffractograms with an estimated number of layers, as well as the surface area of graphitic carbon samples.

\begin{tabular}{|c|c|c|c|c|c|}
\hline \multirow{2}{*}{ Sample } & \multicolumn{2}{|c|}{ Intensity ratio } & \multirow{2}{*}{$\begin{array}{l}\text { FWHM } \\
(002) /^{\circ}\end{array}$} & \multirow{2}{*}{$\begin{array}{l}\text { Approx. } \\
\text { layers* }\end{array}$} & \multirow{2}{*}{$\begin{array}{l}\text { BET surface } \\
\text { area } / \mathrm{m}^{2} \mathrm{~g}^{-1}\end{array}$} \\
\hline & $(002) /(100)$ & $(004) /(100)$ & & & \\
\hline $\begin{array}{l}\text { Natural graphite } \\
\text { flakes }\end{array}$ & 3225.2 & 144.7 & 0.254 & $270-664$ & $1.0 \pm 0.1$ \\
\hline $\begin{array}{l}\text { Graphite powder } \\
\text { (Sigma-Aldrich) }\end{array}$ & 136.3 & 4.8 & 0.331 & $136-400$ & $8.1 \pm 0.1$ \\
\hline $\begin{array}{l}\text { Kaylaw graphite } \\
\text { powder }\end{array}$ & 76.0 & 2.7 & 0.414 & $107-207$ & $25.8 \pm 0.1$ \\
\hline $\begin{array}{l}\text { Exfoliated graphite } \\
\text { (ex Kaylaw) }\end{array}$ & 22.1 & 1.0 & 0.658 & $58-100$ & $47.8 \pm 0.2$ \\
\hline
\end{tabular}

*Estimation of the average number of layers from the crystallite size obtained via Rietveld refinement of the (002) reflection with two graphite structures.

Exfoliation of Kaylaw graphite powder via ultrasonication in NMP27,28 and size selection via centrifugation ${ }^{29}$ almost doubled the surface area of the graphite phase 
while decreasing the calculated average range in the number of layers accordingly by approximately 50\% (Table 2). However, the yield upon ultrasonication in fresh, anhydrous NMP and size-selective separation of the fraction of thicker graphite via centrifugation is only $5 \mathrm{wt} . \%$ and decreases with the number of recycling steps of the valuable solvent NMP, presumably due to contamination with water from the ambient atmosphere. ${ }^{28}$ Analysis of exfoliated graphite (EG) by means of XRD showed decreased ratios of the interplanar reflections relative to the intraplanar reflections confirming a decreased thickness upon exfoliation. Less than 100 layers were estimated from the analysis of the (002) diffraction (Table 2). The actual stacking number may be even lower due to partial re-stacking of thinner few-layer graphite (Figure S.2) during the drying procedure, ${ }^{24}$ which is also indicated by the shift to lower diffraction angles due to an increased d-spacing of imperfect stackings.

Raman spectra of carbon materials show only few prominent features. However, Raman spectroscopy has become the standard for characterisation and analysis in carbon research. ${ }^{52}$ The $G$ band ( $G$ for graphite) at approximately $1560 \mathrm{~cm}^{-1}$ for visual excitation is typically assigned to the bond stretching of all pairs of $\mathrm{sp}^{2}$-hybridised carbon atoms in rings or chains and is therefore associated to the graphitic structure of pristine graphite powder. ${ }^{52-54}$ The breathing modes of $\mathrm{sp}^{2}$ atoms in carbon hexagons result in the $\mathrm{D}$ band ( $\mathrm{D}$ for disorder or defect) at $1360 \mathrm{~cm}^{-1,52-55}$ which is not observed in the centre of a graphite sheets, yet on the edge. ${ }^{56}$ In regard to carbon as catalyst support, the $\mathrm{D}$ band generally represents the functionalisation of the carbon carrier due to induced defects. The Raman spectra of Kaylaw graphite powder before and after exfoliation display similar features (Figure 2). The ratio of the intensities of the $\mathrm{D}$ band over the $G$ band $\left(I_{D} / I_{G}\right)$ is inversely proportional to the in-plane crystallite size $\left(L_{a}\right)^{43,55,57}$ and increases from 0.18 to 0.29 , i.e. exfoliation induces a higher defect concentration as more carbon atoms are associated to the edge for a smaller in-plane dimension. ${ }^{55}$ An estimation of the in-plane crystallite size with the intensity ratio of both prominent bands according to Cançado et al. ${ }^{43}$ (Equation 4) results in $106.8 \mathrm{~nm}$ for pristine Kaylaw graphite powder and $66.3 \mathrm{~nm}$ after exfoliation. No significant differences can be identified in the $2 \mathrm{D}$ band at approximately $2710 \mathrm{~cm}^{-1},{ }^{56}$ formerly referred to as $\mathrm{G}^{\prime}$ band. ${ }^{58}$ The broad peak of said band indicates a 1:1 intensity ratio of the $2 \mathrm{D}_{1}$ and $2 \mathrm{D}_{2}$ band suggesting relatively thin graphite flakes, ${ }^{52}$ which is supported by XRD analysis (Table 2).

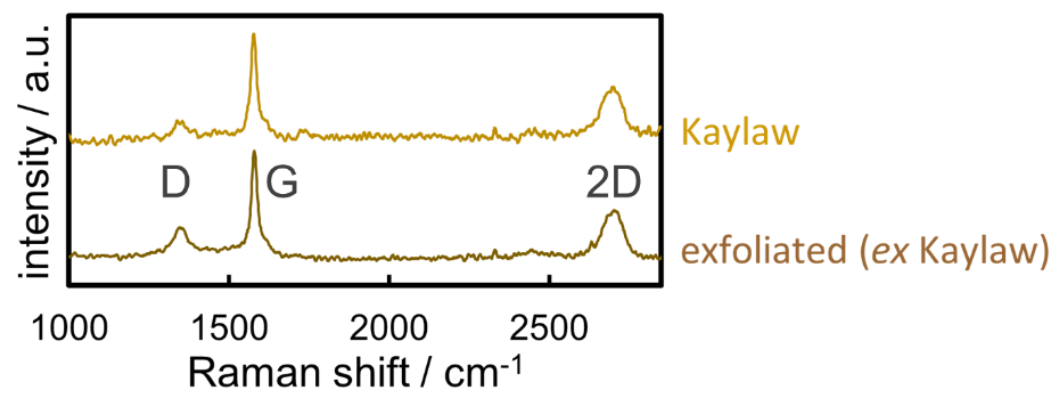


Figure 2 Raman spectra of pristine and exfoliated Kaylaw graphite powder displaying carbon characteristic bands.

Characterisation of EG and the parent Kaylaw graphite powder under an inert $\mathrm{N}_{2}$ atmosphere by means of TGA (Figure S.3a) strongly supports the conclusions drawn from the analysis of Raman spectra. The weight loss during exposure to increased temperatures can be assigned to the decomposition of particular functional groups on the surface of carbon materials. ${ }^{15,44,59}$ When normalised to the available surface area as determined via BET analysis, EG and Kaylaw graphite powder show comparably low concentrations of functional groups (Figure S.3a). No more functional groups decompose on the graphitic samples at temperatures exceeding $500{ }^{\circ} \mathrm{C}$. The low degree of functionalisation (weight loss: $0.06 \%\left(\mathrm{~m}^{2} / \mathrm{g}\right)^{-1}$ ) can be demonstrated when compared to the normalised weight loss of solid carbon spheres, ${ }^{60}$ which is more than 5 times the loss of Kaylaw graphite powder at $500{ }^{\circ} \mathrm{C}$. In addition, significant amounts of functional groups and/or amorphous carbon are converted at increased temperatures. The weight loss due to decomposition of functional groups, when normalised to the BET surface area, is also lower than reported for as-grown multiwalled carbon nanotubes (CNTs; $\left.0.11 \%\left(\mathrm{~m}^{2} / \mathrm{g}\right)^{-1}\right) .{ }^{59}$ The weight fraction in said material has been demonstrated to increase to approximately $0.22 \%\left(\mathrm{~m}^{2} / \mathrm{g}\right)^{-1}$ by applying an acidic treatment in $\mathrm{HNO}_{3} .{ }^{59}$ In the present study, TGA was also applied to verify the chemical purity of Kaylaw graphite powder and resulted in a full conversion upon oxidation in air at $900{ }^{\circ} \mathrm{C}$ (Figure S.3b).

\subsection{Characterisation and supporting of $\mathrm{Co}_{3} \mathrm{O}_{4}$ nanoparticles}

Four distinct sizes of monodisperse $\mathrm{Co}_{3} \mathrm{O}_{4}$ crystallites were synthesised via the benzyl alcohol-route. ${ }^{9,40,41}$ TEM analysis reveals the high dispersibility of the nanoparticles which are nicely spread out over the grid (Figure 3a-d). All four sizes show narrow size distributions (Figure 3e) with relative standard deviations ranging from 16 to 29\% (Table 3). Analysis by means of XRD confirmed the successful preparation of singlephase $\mathrm{Co}_{3} \mathrm{O}_{4}$ nanoparticles of high crystallinity (Figure 3f). Rietveld refinement results in volume-mean crystallite sizes of $2.8,3.5,4.9$, and $7.6 \mathrm{~nm}$ (denoted Co_2.8, Co_3.5, Co_4.9, and Co_7.6, respectively), which were confirmed via application of the Scherrer equation ${ }^{48}$ and TEM analysis (Table 3 ). 

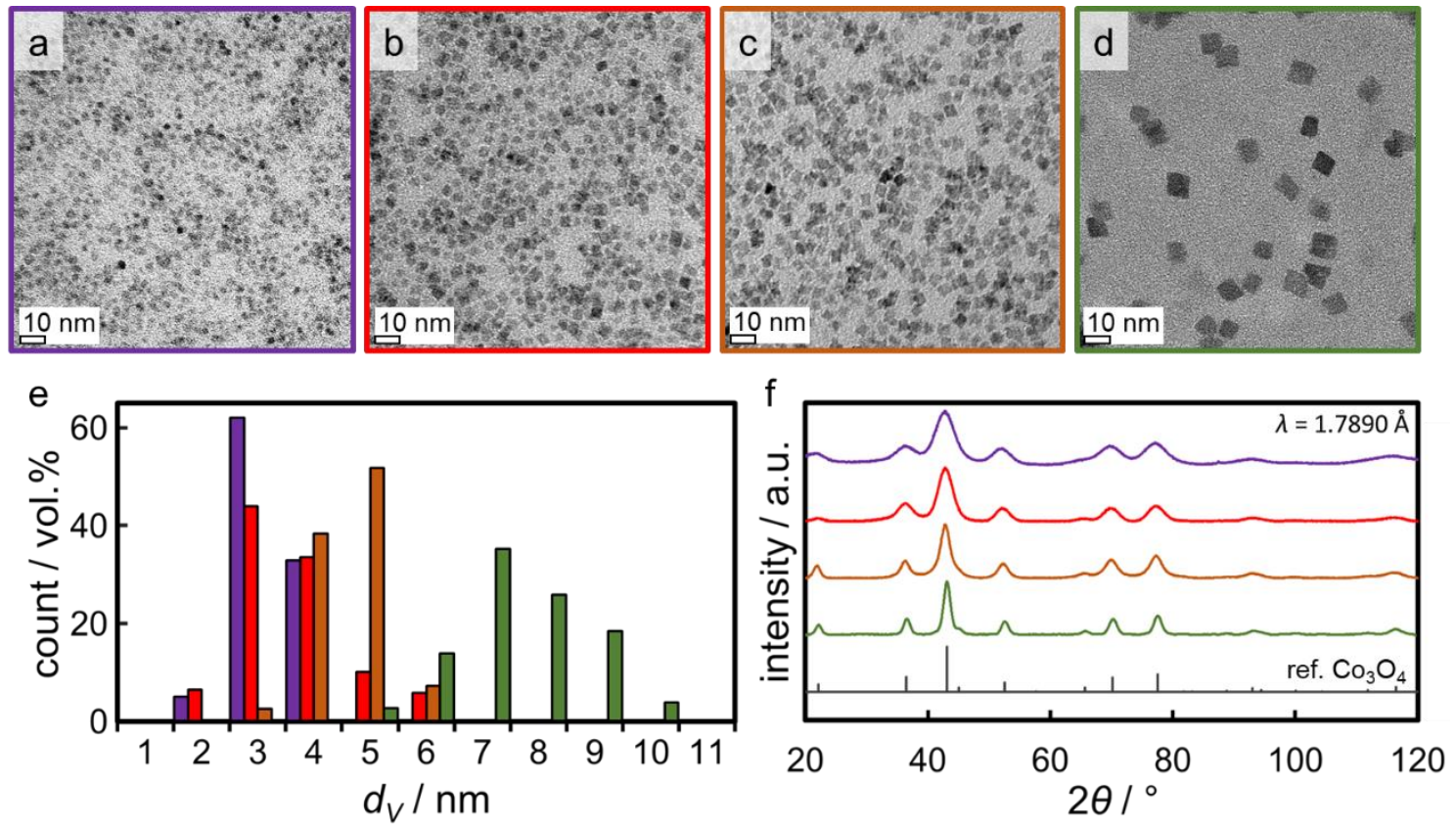

Figure 3 (a-d) Transmission electron micrographs of the $\mathrm{CO}_{3} \mathrm{O}_{4}$ nanoparticles obtained in synthesis Co_2.8, Co_3.5, Co_4.9, and Co_7.6, respectively, together with (e) the particular volume-based size distributions and ( $f$ ) X-ray diffractograms with a reference pattern for $\mathrm{CO}_{3} \mathrm{O}_{4}$. In part reproduced from Ref. ${ }^{9}$ with permission from The Royal Society of Chemistry.

Table 3 Obtained volume-mean crystallite sizes of synthesised $\mathrm{CO}_{3} \mathrm{O}_{4}$ nanoparticles as calculated from X-ray diffraction patterns via Scherrer equation and Rietveld refinement, as well as volume-mean crystallite sizes with relative standard deviations and number-mean crystallite sizes from analysis of transmission electron micrographs.

\begin{tabular}{llllll}
\hline Sample & $d_{V, X R D, R} / \mathrm{nm}$ & $d_{V, X R D, S} / \mathrm{nm}$ & $d_{V, \text { TEM }} / \mathrm{nm}$ & $\sigma_{V, \text { TEM }} / \%$ & $d_{n, \text { TEM }} / \mathrm{nm}$ \\
\hline Co_2.8 & 2.8 & 3.0 & 3.2 & 20.5 & 3.0 \\
Co_3.5 & 3.5 & 4.1 & 3.6 & 28.6 & 3.0 \\
Co_4.9 & 4.9 & 5.0 & 4.6 & 16.2 & 4.3 \\
Co_7.6 & 7.6 & 6.5 & 7.6 & 16.3 & 7.1 \\
\hline
\end{tabular}

The separately synthesised nanoparticles were supported on EG in order to assess the suitability of such novel model systems for the isolated characterisation of metallic Co crystallites. The physicochemical surface properties of the support can be expected to induce differently pronounced interactions with the nanoparticles, which affect their dispersion over the support during the supporting procedure. Three model catalysts with a targeted 5 wt.\% Co loading were prepared and analysed by means of TGA and TEM. TGA resulted in actual Co loadings in the range of 4.4 to 5.0 wt.\% (Figure S.4), i.e. the three samples showed a similar concentration of Co. The thin flakes of exfoliated graphite and the large density difference between the support material and the $\mathrm{Co}_{3} \mathrm{O}_{4}$ 
nanoparticles allow for a clear distinction between the nanoparticles and the carrier (Figure 4). Comparison of the parent catalysts consisting of the $\mathrm{Co}_{3} \mathrm{O}_{4}$ nanoparticles Co_2.8, Co_4.9, and Co7.6 supported on EG demonstrates the different dispersion levels of the oxidic cobalt phase over the available surface area of the carrier. The larger nanoparticles in the $5 \mathrm{wt} . \%$ Co_7.6/EG and Co_4.9/EG samples are well distributed and only small aggregates are identified. A high dispersion over the surface area of the support was achieved for the smaller nanoparticles, but the surface of the EG appears to be crowded due to the large number of small crystallites required for the $5 \mathrm{wt} . \%$ loading.

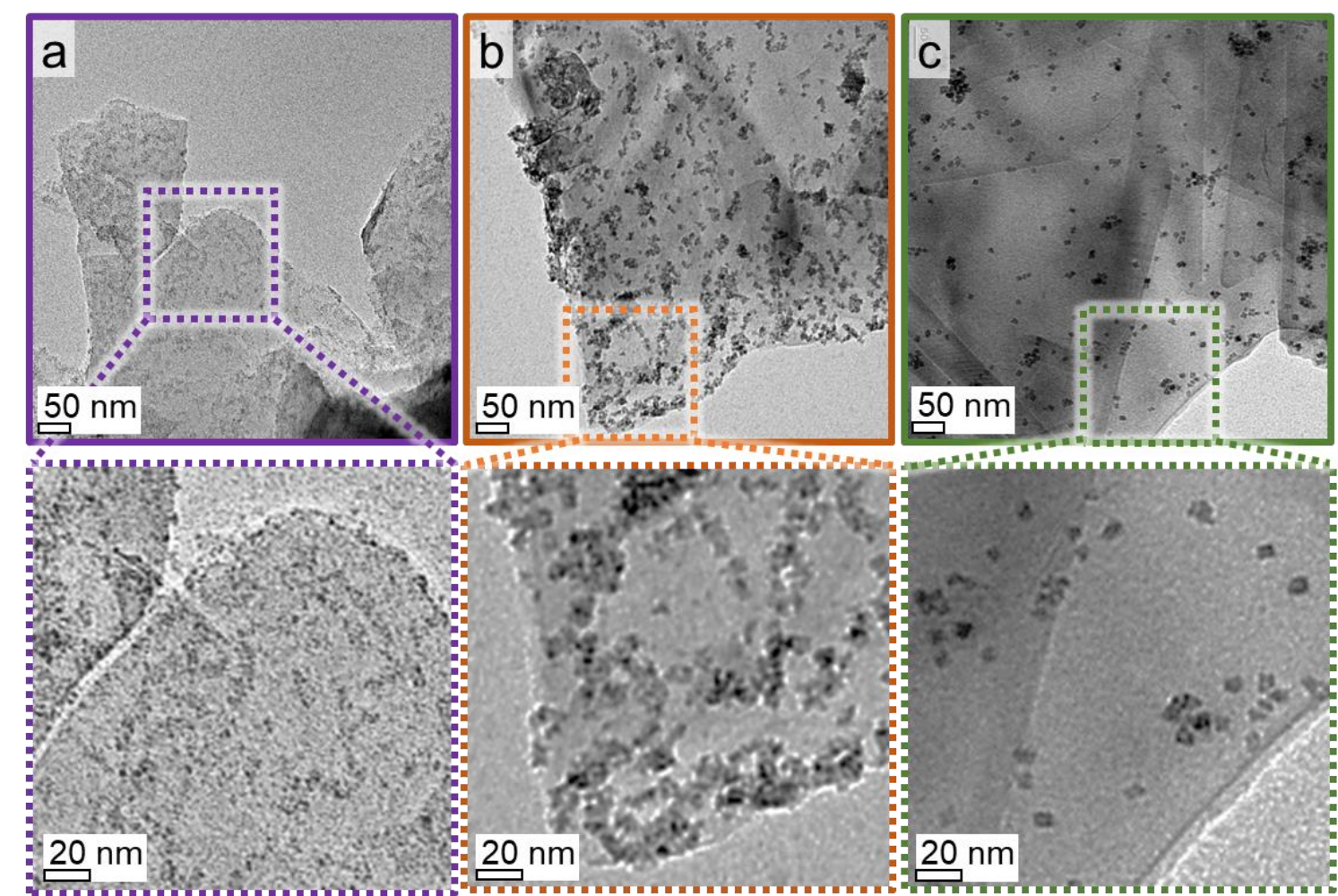

Figure 4 Transmission electron micrographs of $5 \mathrm{wt} . \%$ (a) Co_2.8, (b) Co_4.9, and (c) Co_7.6 on exfoliated graphite with magnified insets.

In order to reduce the extent of agglomeration of the well-defined $\mathrm{Co}_{3} \mathrm{O}_{4}$ crystallites, the loading of Co was reduced to $1 \mathrm{wt} . \%$ and EG has been decorated with several sizes of $\mathrm{Co}_{3} \mathrm{O}_{4}$ nanoparticles $\left(2.8,3.5\right.$, and $7.6 \mathrm{~nm}$ denoted $\mathrm{Co}_{-} 2.8, \mathrm{Co}_{-} 3.5$, and $\mathrm{Co}_{-} 7.5$, respectively). TGA resulted in Co loadings of 0.9 to $1.1 \mathrm{wt} . \%$ (Figure S.5). The samples comprising the smaller sizes show a high dispersion of nanoparticles over the support surface (Figure 5). In contrast, some small aggregates were identified by means of TEM in the samples comprising the larger nanoparticles. However, the fraction of welldispersed isolated crystallites increased drastically compared to the sample with a 5 wt.\% Co loading. 


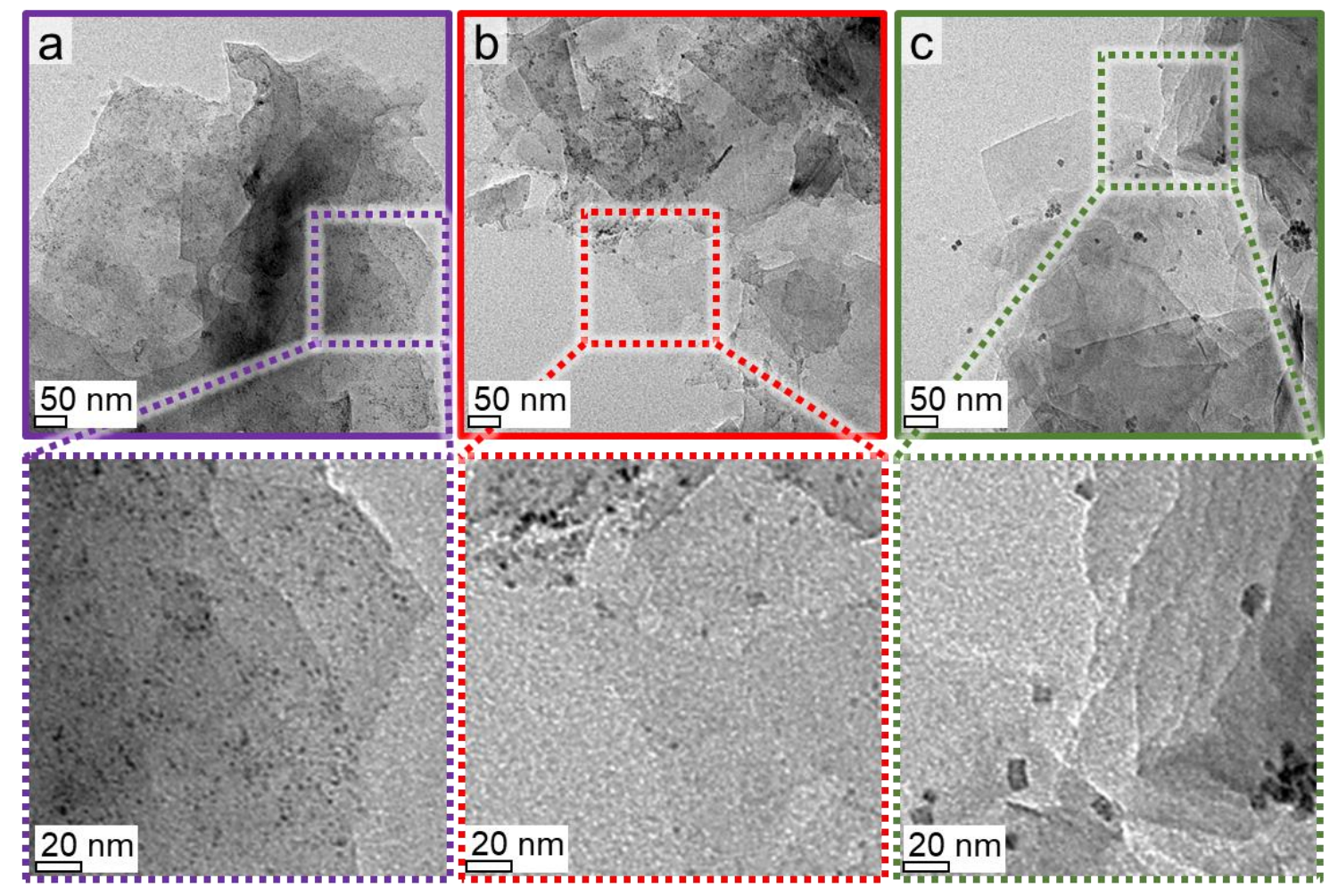

Figure 5 Transmission electron micrographs of $1 \mathrm{wt. \%}$ (a) Co_2.8, (b) Co_3.5, and (c) Co_7.6 on exfoliated graphite with magnified insets.

\section{Conclusions}

Graphitic carbon materials were characterised and tested for their suitability as support material for cobaltous nanoparticles in model catalyst systems. When compared to pristine graphite powder, exfoliated graphite was shown to provide a significantly increased surface area allowing for a high dispersion of nanoparticles over the support surface. However, a cobalt loading as low as $1 \mathrm{wt} . \%$ is required in order to minimise the formation of aggregates which may easily sinter during reduction or any other thermal treatment. The developed model catalyst provides great opportunities in order to study isolated $\mathrm{Co}_{3} \mathrm{O}_{4}$ or even metallic cobalt nanoparticles in various applications. Exfoliated graphite as support material further allows for fundamental insight into the physicochemical properties of supported nanoparticles by limiting the influence of the carrier material.

\section{Acknowledgements}

Financial support from the DST-NRF Centre of Excellence in Catalysis ( ${ }^{*}$ change), the University of Cape Town (UCT), and the German Academic Exchange Service (DAAD) is gratefully acknowledged. 


\section{References}

$1 \quad$ E. Iglesia, S. L. Soled and R. A. Fiato, J. Catal., 1992, 137, 212-224.

$2 \emptyset$. Borg, S. Eri, E. A. Blekkan, S. Storsæter, H. Wigum, E. Rytter and A. Holmen, J. Catal., 2007, 248, 89-100.

3 S. Lögdberg, M. Boutonnet, J. C. Walmsley, S. Järås, A. Holmen and E. A. Blekkan, Appl. Catal. A Gen., 2011, 393, 109-121.

4 F. Bertella, P. Concepción and A. Martínez, Catal. Today, 2016, 289, 181-191.

$5 \quad$ A. Kogelbauer, J. C. Weber and J. G. Goodwin Jr., Catal. Letters, 1995, 34, 259267.

6 B. Jongsomjit, J. Panpranot and J. G. Goodwin Jr., J. Catal., 2001, 204, 98-109.

7 D. J. Moodley, A. M. Saib, J. van de Loosdrecht, C. A. Welker-Nieuwoudt, B. H. Sigwebela and J. W. Niemantsverdriet, Catal. Today, 2011, 171, 192-200.

8 N. E. Tsakoumis, J. C. Walmsley, M. Rønning, W. van Beek, E. Rytter and A. Holmen, J. Am. Chem. Soc., 2017, 139, 3706-3715.

9 M. Wolf, H. Kotzé, N. Fischer and M. Claeys, Faraday Discuss., 2017, 197, $243-$ 268.

10 S. J. Tauster, S. C. Fung, R. T. K. Baker and J. A. Horsley, Science (80-. )., 1981, 21, $1121-1125$.

11 S. J. Tauster, Acc. Chem. Res., 1987, 20, 389-394.

12 N. Fischer, M. Minnermann, M. Baeumer, E. van Steen and M. Claeys, Catal. Letters, 2012, 142, 830-837.

13 G. L. Haller and D. E. Resasco, Adv. Catal., 1989, 36, 173-235.

14 M. Wolf, E. K. Gibson, E. J. Olivier, J. H. Neethling, C. R. A. Catlow, N. Fischer and M. Claeys, ACS Catal., 2019, 9, 4902-4918.

15 J. L. Figueiredo, M. F. R. Pereira, M. M. A. Freitas and J. J. M. Órfão, Carbon N. Y., 1999, 37, 1379-1389.

16 N. Karousis, N. Tagmatarchis and D. Tasis, Chem. Rev., 2010, 110, 5366-5397.

17 D. S. Su, S. Perathoner and G. Centi, Chem. Rev., 2013, 113, 5782-5816.

18 J. van de Loosdrecht, M. Datt and J. L. Visagie, Top. Catal., 2014, 57, 430-436.

19 G. L. Bezemer, J. H. Bitter, H. P. C. E. Kuipers, H. Oosterbeek, J. E. Holewijn, X. Xu, F. Kapteijn, A. J. van Dillen and K. P. de Jong, J. Am. Chem. Soc., 2006, 128, 3956-3964.

20 M. Trépanier, A. K. Dalai and N. Abatzoglou, Appl. Catal. A Gen., 2010, 374, 7986.

$21 \quad$ F. Rodríguez-Reinoso, Carbon N. Y., 1998, 36, 159-175.

22 M. Wolf, B. K. Mutuma, N. J. Coville, N. Fischer and M. Claeys, ACS Catal., 2018, 8, 3985-3989.

23 M. Cai, D. Thorpe, D. H. Adamson and H. C. Schniepp, J. Mater. Chem., 2012, 22, 24992-25002.

24 V. Singh, D. Joung, L. Zhai, S. Das, S. I. Khondaker and S. Seal, Prog. Mater. Sci., 2011, 56, 1178-1271.

25 J. N. Coleman, M. Lotya, A. O’Neill, S. D. Bergin, P. J. King, U. Khan, K. Young, A. Gaucher, S. De, R. J. Smith, I. V. Shvets, S. K. Arora, G. Stanton, H.-Y. Kim, K. Lee, G. T. Kim, G. S. Duesberg, T. Hallam, J. J. Boland, J. J. Wang, J. F. Donegan, J. C. Grunlan, G. Moriarty, A. Shmeliov, R. J. Nicholls, J. M. Perkins, E. M. Grieveson, K. Theuwissen, D. W. McComb, P. D. Nellist and V. Nicolosi, Science 
(80-. )., 2011, 331, 568-571.

W. Zhao, M. Fang, F. Wu, H. Wu, L. Wang and G. Chen, J. Mater. Chem., 2010, 20, 5817-5819.

Y. Hernandez, V. Nicolosi, M. Lotya, F. M. Blighe, Z. Sun, S. De, I. T. McGovern, B. Holland, M. Byrne, Y. K. Gun'Ko, J. J. Boland, P. Niraj, G. Duesberg, S.

Krishnamurthy, R. Goodhue, J. Hutchison, V. Scardaci, A. C. Ferrari and J. N. Coleman, Nat. Nanotechnol., 2008, 3, 563-568.

U. Khan, A. O’Neill, M. Lotya, S. De and J. N. Coleman, Small, 2010, 6, 864-871.

U. Khan, A. O’Neill, H. Porwal, P. May, K. Nawaz and J. N. Coleman, Carbon N. Y., 2012, 50, 470-475.

30 W. S. Hummers Jr. and R. E. Offeman, J. Am. Chem. Soc., 1958, 80, 1339.

31 J. I. Paredes, S. Villar-Rodil, A. Martínez-Alonso and J. M. D. Tascón, Langmuir, 2008, 24, 10560-10564.

32 Y. Si and E. T. Samulski, Nano Lett., 2008, 8, 1679-1682.

33 B. F. Machado and P. Serp, Catal. Sci. Technol., 2012, 2, 54-75.

34 S. Stankovich, D. A. Dikin, G. H. B. Dommett, K. M. Kohlhaas, E. J. Zimney, E. A. Stach, R. D. Piner, S. T. Nguyen and R. S. Ruoff, Nature, 2006, 442, 282-286.

35 W. Gao, L. B. Alemany, L. Ci and P. M. Ajayan, Nat. Chem., 2009, 1, 403-408.

36 H. Zhang, C. Lancelot, W. Chu, J. Hong, A. Y. Khodakov, P. A. Chernavskii, J. Zheng and D. Tong, J. Mater. Chem., 2009, 19, 9241-9249.

37 H. Xiong, L. L. Jewell and N. J. Coville, ACS Catal., 2015, 2640-2658.

38 J. R. C. Salgado, R. G. Duarte, L. M. Ilharco, A. M. Botelho do Rego, A. M. Ferraria and M. G. S. Ferreira, Appl. Catal. B Environ., 2011, 102, 496-504. W. Xia, C. Jin, S. Kundu and M. Muhler, Carbon N. Y., 2009, 47, 919-922.

40 N. Shi, W. Cheng, H. Zhou, T. Fan and M. Niederberger, Chem. Commun., 2015, 51, 1338-1340.

41 M. Wolf, N. Fischer and M. Claeys, Mater. Chem. Phys., 2018, 213, 305-312.

42 W. Rasband, ImageJ 1.51a, National Institute of Mental Health, Bethesda, USA, 2016.

43 L. G. Cançado, K. Takai, T. Enoki, M. Endo, Y. A. Kim, H. Mizusaki, A. Jorio, L. N. Coelho, R. Magalhães-Paniago and M. A. Pimenta, Appl. Phys. Lett., 2006, 88, $12-14$.

44 S. A. El-Khodary, G. M. El-Enany, M. El-Okr and M. Ibrahim, Electrochim. Acta, 2014, 150, 269-278.

45 M. Chen, B. Hallstedt and L. J. Gauckler, J. Phase Equilibria, 2003, 24, 212-227.

46 ICDD, PDF-2 Release 2008 (Database), International Centre for Diffraction Data, Newtown Square, USA, 2008.

47 A. A. Coelho, J. Appl. Crystallogr., 2003, 36, 86-95.

48 P. Scherrer, Nachrichten von der Gesellschaft der Wissenschaften zu Göttingen, Math. Klasse, 1918, 98-100.

49 G. Bergeret and P. Gallezot, in Handbook of Heterogeneous Catalysis, eds. G. Ertl, H. Knözinger, F. Schüth and J. Weitkamp, Wiley-VCH, Weinheim, Germany, 2nd edn., 2008, pp. 738-765.

50 H. Fujimoto, Carbon N. Y., 2003, 41, 1585-1592.

51 C. N. R. Rao, K. Biswas, K. S. Subrahmanyam and A. Govindaraj, J. Mater. Chem., 2009, 19, 2457-2469.

52 A. C. Ferrari, Solid State Commun., 2007, 143, 47-57. 
53 M. S. Dresselhaus, A. Jorio, A. G. Souza Filho and R. Saito, Philos. Trans. R. Soc. London A Math. Phys. Eng. Sci., 2010, 368, 5355-5377.

54 C. Castiglioni, F. Negri, M. Rigolio and G. Zerbi, J. Chem. Phys., 2001, 115, 37693778.

55 F. Tuinstra and J. L. Koenig, J. Chem. Phys., 1970, 53, 1126-1130.

56 A. C. Ferrari, J. C. Meyer, V. Scardaci, C. Casiraghi, M. Lazzeri, F. Mauri, S. Piscanec, D. Jiang, K. S. Novoselov, S. Roth and A. K. Geim, Phys. Rev. Lett., 2006, 97, 1-4.

57 D. S. Knight and W. B. White, J. Mater. Res., 1989, 4, 385-393.

58 R. P. Vidano, D. B. Fischbach, L. J. Willis and T. M. Loehr, Solid State Commun., 1981, 39, 341-344.

59 M. A. M. Motchelaho, H. Xiong, M. Moyo, L. L. Jewell and N. J. Coville, J. Mol. Catal. A Chem., 2011, 335, 189-198.

60 B. K. Mutuma, B. J. Matsoso, K. Ranganathan, J. M. Keartland, D. Wamwangi and N. J. Coville, RSC Adv., 2017, 7, 21187-21195. 


\section{Employing exfoliated graphite as novel support material for heterogeneous model catalysts}

Moritz Wolf, ${ }^{\dagger, 1}$ Nico Fischer, ${ }^{\dagger}$ and Michael Claeys*, ${ }^{*}$

${ }^{\dagger}$ Catalysis Institute and DST-NRF Centre of Excellence in Catalysis c*change, Department of Chemical Engineering, University of Cape Town, Rondebosch 7701, South Africa.

\section{Supplementary Figures}
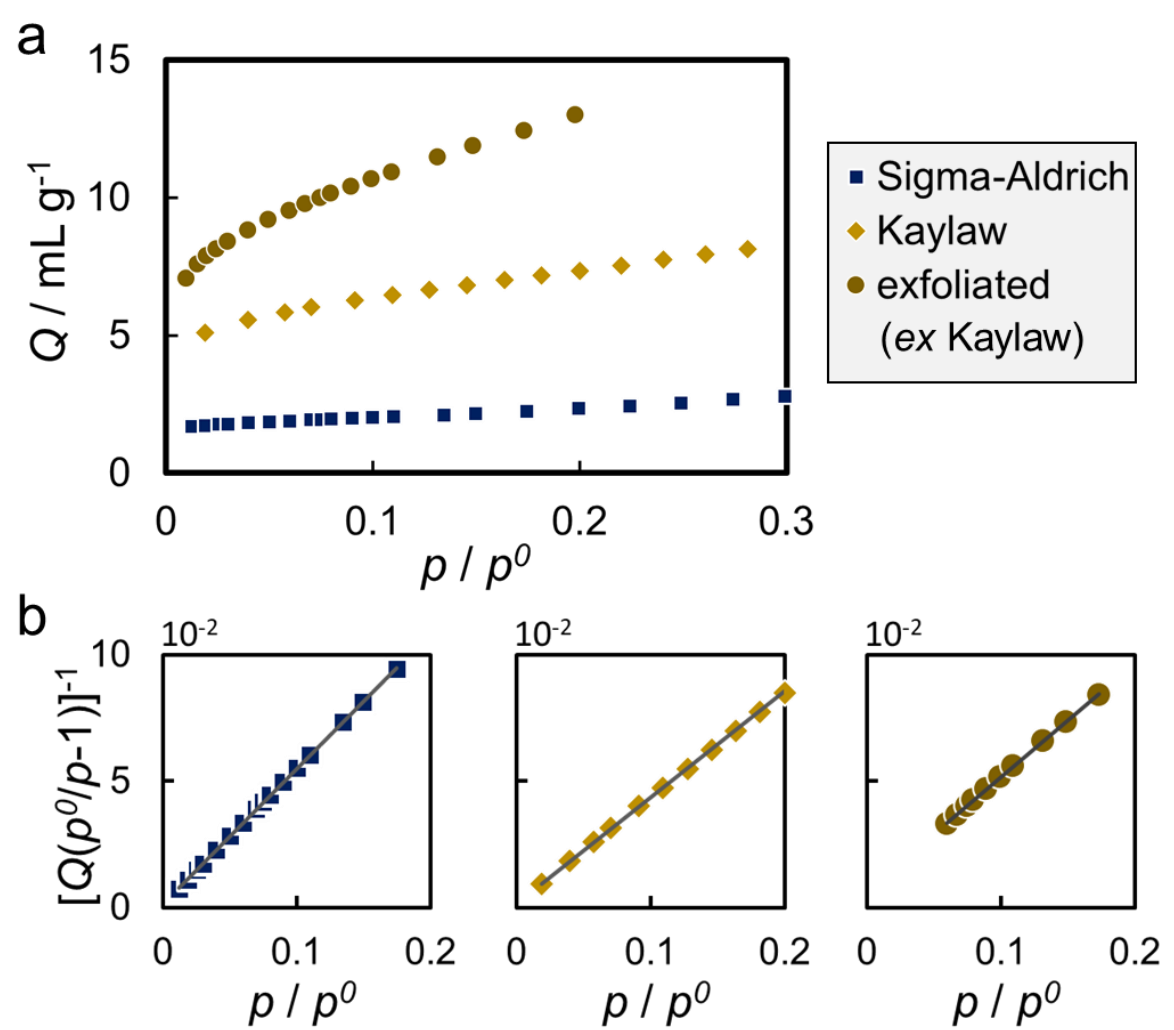

Figure S.1 (a) Nitrogen adsorption isotherms and (b) fits to the Brunauer-EmmettTeller equation of studied graphite powders.

\footnotetext{
${ }^{1}$ Present address: Institute of Chemical Reaction Engineering, University of Erlangen-Nuremberg, 91058 Erlangen, Germany
} 

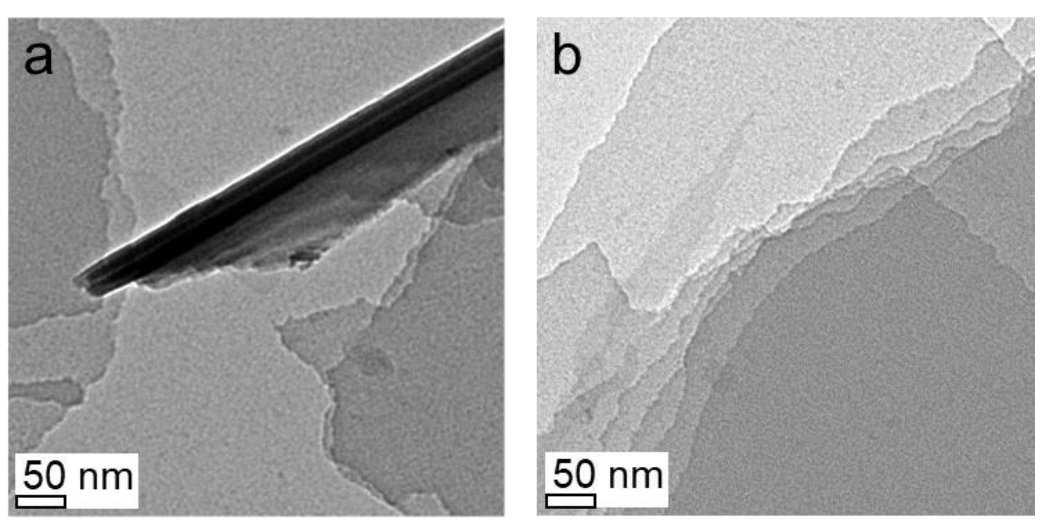

Figure S.2 Transmission electron micrographs of exfoliated graphite with a flake in (a) side view and (b) top view.

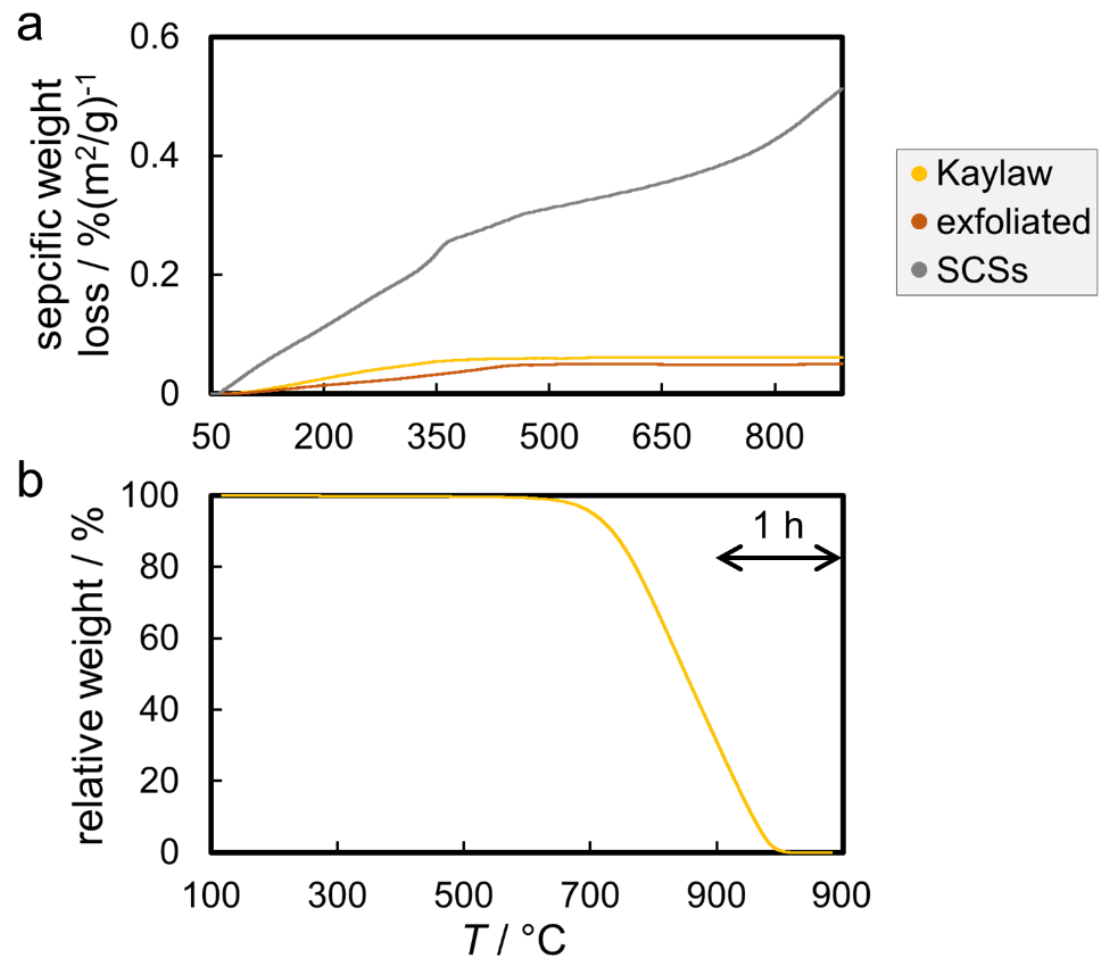

Figure S.3 Thermogravimetric analysis of (a) Kaylaw graphite powder, exfoliated Kaylaw graphite powder, as well as solid carbon spheres (SCSs) ${ }^{1}$ as a reference material for functionalised carbon under an inert $\mathrm{N}_{2}$ atmosphere during a temperature ramp from 50 to $900{ }^{\circ} \mathrm{C}$ at $5^{\circ} \mathrm{C} \mathrm{min}-1$, as well as (b) combustion of Kaylaw graphite powder in air during a temperature ramp from 120 to $900{ }^{\circ} \mathrm{C}$ at $10{ }^{\circ} \mathrm{C} \mathrm{min}-1$ with a 60 min holding time at $900{ }^{\circ} \mathrm{C}$. 


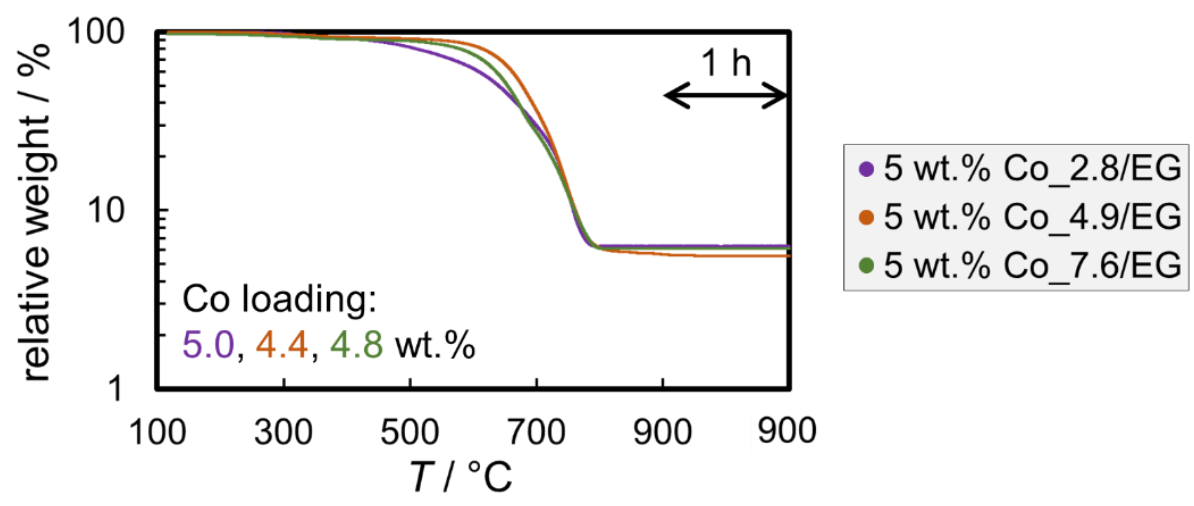

Figure S.4 Thermogravimetric analysis of $5 \mathrm{wt} . \% \mathrm{Co} / \mathrm{EG}$ model catalysts in air during a temperature ramp from 120 to $900{ }^{\circ} \mathrm{C}$ at $10{ }^{\circ} \mathrm{C} \mathrm{min}-1$ with a 60 min holding time at $900{ }^{\circ} \mathrm{C}$. Note that the residual weight corresponds to the mass of CoO.

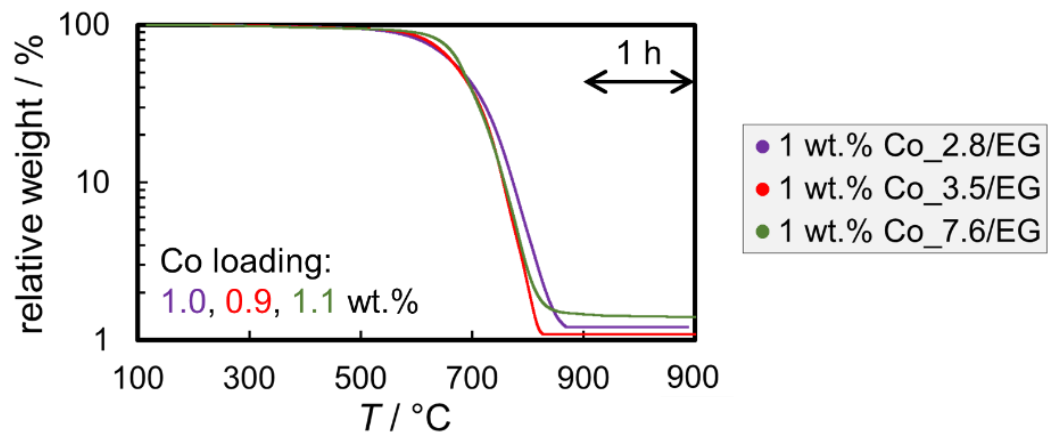

Figure S.5 Thermogravimetric analysis of $5 \mathrm{wt}$.\% Co/EG model catalysts in air during a temperature ramp from 120 to $900{ }^{\circ} \mathrm{C}$ at $10^{\circ} \mathrm{C} \mathrm{min}-1$ with a 60 min holding time at $900{ }^{\circ} \mathrm{C}$. Note that the residual weight corresponds to the mass of CoO.

\section{References}

1 B. K. Mutuma, B. J. Matsoso, K. Ranganathan, J. M. Keartland, D. Wamwangi and N. J. Coville, RSC Adv., 2017, 7, 21187-21195. 
